\title{
EDITORIAL du Numéro spécial J3EA dédié aux JPCNFM'2018 : Formation en microélectronique et nanotechnologie orientée vers le savoir- faire innovant destiné à un environnement numérique
}

\author{
O. Bonnaud ${ }^{\mathrm{a}, \mathrm{b}}$, H. Lhermite ${ }^{\mathrm{a}, \mathrm{c}}$, L. Pichon ${ }^{\mathrm{a}, \mathrm{c}}$, \\ a Dept. Microcapteurs et Microélectronique, IETR, Université de Rennes 1, Rennes, France \\ ${ }^{\mathrm{b}}$ GIP-CNFM, Grenoble, France \\ ${ }^{\mathrm{c}}$ Pôle CNFM de Rennes (CCMO), Université de Rennes 1, Rennes, France \\ Contacts email : olivier.bonnaud@univ-rennes1.fr, lorraine.chagoya@grenoble-inp.fr
}

Après une 14ème édition spéciale organisée à Montpellier à l'occasion des 30 ans des Services Nationaux, ces 15èmes Journées Pédagogiques du GIP-CNFM (1) ont retrouvé leur cadre «traditionnel » au Palais du Grand Large à Saint-Malo. C'est donc le pôle CNFM de Rennes, le CCMO (2), qui a repris en main l'organisation de ces Journées, qui sont un moment important pour le GIP-CNFM depuis 1990.

L'objectif de ces 15èmes Journées Pédagogiques du CNFM a été, dans le même esprit que les éditions précédentes, d'encourager les échanges à caractère pédagogique entre enseignants de notre communauté et d'inciter à l'innovation adaptée aux nouveaux besoins socioéconomiques dans un contexte d'évolution vers une société numérique.

Les éditions précédentes ont prouvé tout l'intérêt de ces journées, d'une part pour les enseignants qui souhaitent faire évoluer leurs enseignements, et d'autre part pour les syndicats professionnels qui s'impliquent dans les orientations de l'adaptation des enseignements aux nouvelles technologies appliquées par l'industrie.

Les thématiques concernent aussi bien la technologie, la conception, le test ou la caractérisation associés aux performances et innovations des circuits micro- et nanoélectroniques, VLSI, de puissance ou de grande surface, ou encore des circuits hybrides, hyperfréquences ou pour télécommunications, des SOC (Systems on Chip), des nouvelles technologies sur supports divers tels que les matériaux flexibles ou plastique (plastronique), voire aux systèmes toujours plus intelligents et connectés.

Une attention particulière a été portée sur des thèmes innovants en accord avec les engagements du GIP-CNFM vis-à-vis du Ministère de l'Enseignement Supérieur de la Recherche et de l'Innovation et du programme IDEFI-FINMINA (3) (Investissements d'Avenir), tels que l'électronique portable, les micro- et nanotechnologies, les technologies avancées impliquant les nouveaux composants sur différents supports, mais aussi les nouvelles approches de conception et réalisation liées à la faible consommation, à l'autonomie, à la sécurité des systèmes matériels, et aux nouveaux usages «smart». L'internet des objets et les systèmes cyber-physiques sont également à l'honneur en tant que marchés porteurs d'emplois pour nos jeunes diplômés.

Par ailleurs, des exposés ont été dédiés aux expériences de sensibilisation de cette discipline vers les lycées et collèges qui s'intègrent dans la politique du GIP-CNFM et du Ministère de l'Education et qui répond aux besoins des entreprises. Ces actions, qui sont l'un des points forts de la stratégie du CNFM, ont un impact important sur le futur de la discipline.

Ces journées ont aussi été l'occasion de confronter les aspects pédagogiques aux besoins de nos partenaires industriels de la microélectronique par leur contribution dans les sessions plénières. Comme lors des éditions précédentes, les représentants de la profession au sein d'ACSIEL Alliance Electronique (4), dont Le Président également nouveau président du GIPCNFM, ont participé aux journées pédagogiques et ont apporté à la communauté académique

This is an Open Access article distributed under the terms of the Creative Commons Attribution License (http://creativecommons.org/licenses/by/4.0), which permits unrestricted use, distribution, and reproduction in any medium, provided the original work is properly cited. 
leur vision industrielle dans un nouveau contexte de Filière Electronique Française reconnue par la Direction Générale des Entreprises. Le partenariat monde académique / monde industriel du domaine va se trouver renforcé et de futures actions communes sont déjà engagées au sein du Comité Stratégique de Filière qui comporte des représentants d'ACSIEL Alliance Electronique et du GIP-CNFM. En lien avec ce partenariat, la formation continue et tout au long de la vie ont constitué un sujet de réflexion important puisqu'il va apparaître comme un point stratégique de la filière qui nécessitera des nouvelles compétences et savoir-faire des futurs diplômés et des professionnels en exercice.

Comme pour les éditions précédentes, les actes de ces journées témoignent de l'investissement de l'ensemble des collègues utilisateurs des plateformes du réseau national réparties dans les douze pôles, sans lequel le réseau n'aurait pas de sens. Afin d'augmenter l'impact de ces investissements pédagogiques récents du réseau, comme lors des éditions précédentes, les actes sont publiés, après sélection et relecture, dans ce numéro spécial du journal national en ligne et à finalité pédagogique, le J3EA au sein d'EDP Sciences afin de mieux disséminer les résultats du réseau notamment dans son aspect innovant fortement soutenu par le programme IDEFI et son projet FINMINA.

Cette manifestation organisée par le pôle CNFM de Rennes, le CCMO, a reçu le soutien du GIP-CNFM et du projet IDEFI-FINMINA. La direction du GIP-CNFM a pu organiser la publication de ce numéro spécial grâce à une très forte implication de Lorraine-Chagoya Garzon, assistante de direction du GIP-CNFM.

Les organisateurs, auteurs de cet éditorial, remercient l'ensemble des contributeurs et le comité d'organisation local sans lesquels ce numéro spécial de J3EA ne pourrait exister.

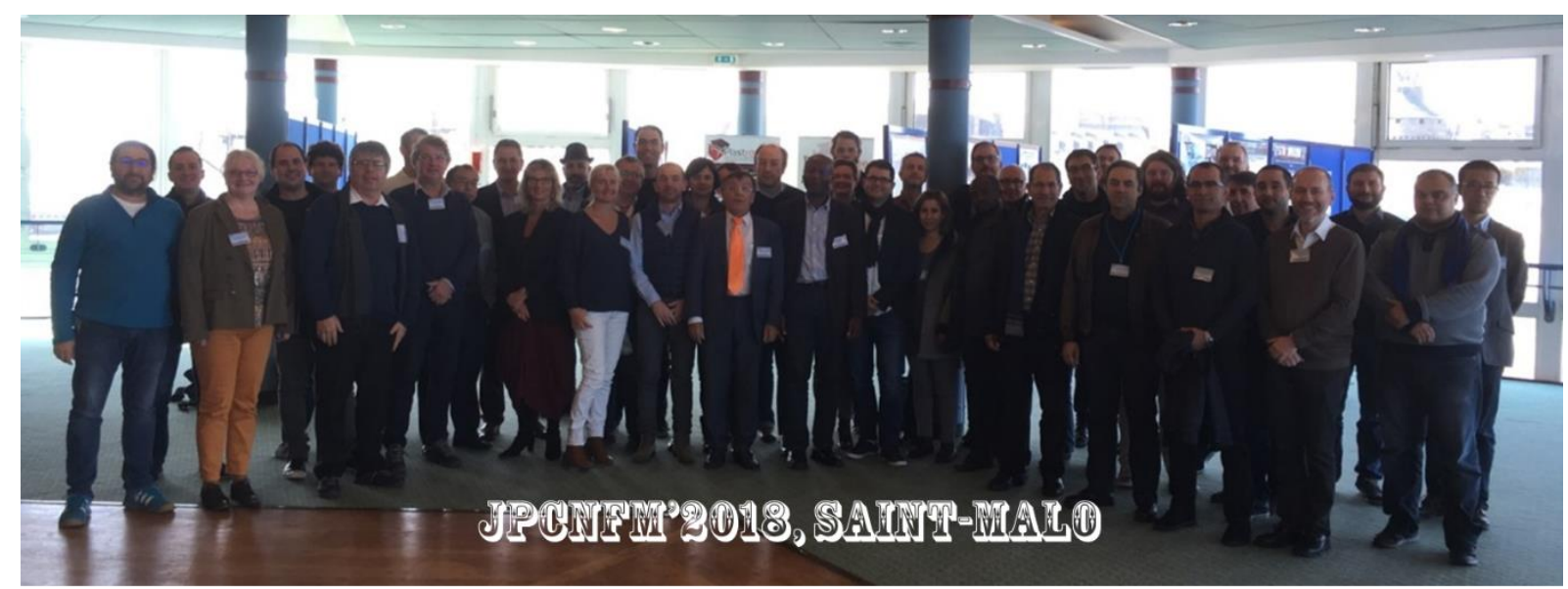

\section{Références}

1. GIP-CNFM: Groupement d'Intérêt Public - Coordination Nationale pour la formation en Microélectronique et en nanotechnologies. Website : http://wwww.cnfm.fr

2. CCMO : Centre Commun de Microélectronique de l'Ouest. Website : http://ccmo.univ-rennes1.fr

3. IDEFI-FINMINA : Initiative d'Excellence - Formation Innovante en MIcroélectronique et Nanotechnologies, ANR-11-IDFI-0017. Website: http://www.cnfm.fr/VersionFrancaise/actualites/ FINMINA.htm

4. ACSIEL Alliance Electronique: website: http://www.acsiel.fr/en-GB/index.aspx 